\title{
Density Fluctuations as Precursors of Crystallization in a Thermoplastic Polyimide
}

\author{
Fernando Ania, Maria Esperanza Cagiao, and Francisco Jose Baltá Calleja \\ Instituto de Estructura de la Materia, CSIC, Serrano 119, 28006 Madrid, Spain
}

(Received January 18, 1999)

\begin{abstract}
The present report is part of a general study aiming to investigate the possible evidence of electron density fluctuations as precursors of crystallization in polymers with different chain stiffness. The present experiments on the thermoplastic polyimide (New-TPI) with varying induction times, studied by small- and wide-angle X-ray scattering (SAXS and WAXS), reveal the onset of long-range ordering prior to crystal growth. Slow crystallization, slightly above $T_{\mathrm{g}}$ indicates the development of well resolved SAXS patterns associated with long range order before the development of crystalline peaks in the WAXS region. The experimental results suggest that density fluctuations control at sufficiently low temperatures the precrystallization mechanisms during the embryonic period.

KEY WORDS Electron Density Fluctuations / Simultaneous Small-Angle X-Ray Scattering and Wide-Angle X-Ray Scattering / Cold Crystallization / New-Thermoplastic Polyimide /
\end{abstract}

The development of crystallinity in the amorphous thermoplastic polyimide (New-TPI) has been studied, in detail, using differential scanning calorimetry, wideand small-angle X-ray scattering (WAXS and SAXS) ${ }^{1,2}$ and microhardness, ${ }^{3}$ by cold crystallization from the amorphous state. Preceding SAXS studies show that during cold crystallization, New-TPI develops a periodic structure with crystals a few molecular repeat-units thick. ${ }^{1,2}$ Time resolved SAXS studies reveal, in addition, that both, long period and lamellar thickness show a decreasing dependence with time. Cold crystallization kinetics from DSC has been described by a single Avrami exponent, $n=3.5 .^{2}$ The study of the crystallization mechanism during the embryonic period of TPI is, however, still missing. The ordering process prior to crystallization has been studied for semirigid ${ }^{4,5}$ and flexible ${ }^{6}$ polymers by means of simultaneous real time WAXS and SAXS. In these systems phase separation has been shown to follow spinodal decomposition. The latter involves two regions of the polymer, one having the appropriate chain conformation for crystallization and the other one which becomes the amorphous regions present in all semicrystalline polymers. Recent morphological studies on poly(ethylene 2,6 dicarboxylate naphthalene) (PEN) support the above view evidencing, during the induction period prior to crystallization, a phase separation into crystallizable and non-crystallizable regions. ${ }^{7}$ If spinodal decomposition prevails, development of the SAXS pattern due to density fluctuation occurs prior to any WAXS from the crystals. This is due to the continuous transformation of the partially ordered phase through slightly more ordered states. ${ }^{8}$

The object of the present report is to extend the above studies aiming to investigate the possible evidence of density fluctuation as precursors of crystallization in New-TPI, polymer with the highest chain stiffness so far investigated.

\section{EXPERIMENTAL}

Amorphous thermoplastic polyimide resin (New-TPI) from Mitsui Chemical Co. was used as supplied, ${ }^{9}$ Two transparent $80 \mu \mathrm{m}$ thick films were packed in $\mathrm{Al}$ foil for the time resolved measurements.

Simultaneous WAXS and SAXS experiments were carried out in a double focusing mirror monochromator camera on the polymer beam line (A2) at HASYLAB (Hamburg, Germany) using synchrotron radiation. Cold crystallization was performed isothermally bringing up each sample rapidly to the crystallization temperature from the glassy state. Scattering patterns were recorded using linear position sensitive detectors, corrected for fluctuations in intensity of the primary beam and background. The amorphous New-TPI samples were treated up to $262,265,267,270,272,275,280,285,287$, and $289^{\circ} \mathrm{C}$, respectively, where the isothermal crystallization process was recorded. At $262^{\circ} \mathrm{C}$, the process was so slow that only the starting part of it could be followed due to our experimental time limitation $(6-8 \mathrm{~h})$. X-Ray accumulation time for each frame was set to $60 \mathrm{~s}$ with a waiting time between two consecutive frames ranging between 0 and $120 \mathrm{~s}$. Further experimental details will be given in a separate paper.

\section{RESULTS AND DISCUSSION}

Figures la and $1 \mathrm{~b}$ show the variation of SAXS and WAXS as a function of time for an isothermal experiment carried out at $T_{\mathrm{c}}=270^{\circ} \mathrm{C}$. In the SAXS experiment the initial time pattern was subtracted from all the others to better observe the appearance of a maximum of scattered intensity at a wave vector $\boldsymbol{q}=2 \pi s \approx 0.37 \mathrm{~nm}^{-1}$ (where $s=2 \sin \theta / \lambda$ and $\lambda=0.150 \mathrm{~nm}$ ). Simultaneous WAXS experiments show the start of crystallization evidenced by the presence of three incipient Bragg maxima for times $t>1260 \mathrm{~s}$. Similar experiments were performed at the other above mentioned temperatures.

Figures $2 \mathrm{a}$ and $2 \mathrm{~b}$ show the variation of, both, the normalized SAXS intensity invariant $Q_{\text {norm }}$ and of the relative degree of crystallinity derived from WAXS data as a function of crystallization time for the various temperatures investigated. $Q_{\text {norm }}$ values, different from zero, are observed from the very early stages of crystallization for all $T_{\mathrm{c}}$ temperatures (data for $T_{\mathrm{c}}=262^{\circ} \mathrm{C}$ 

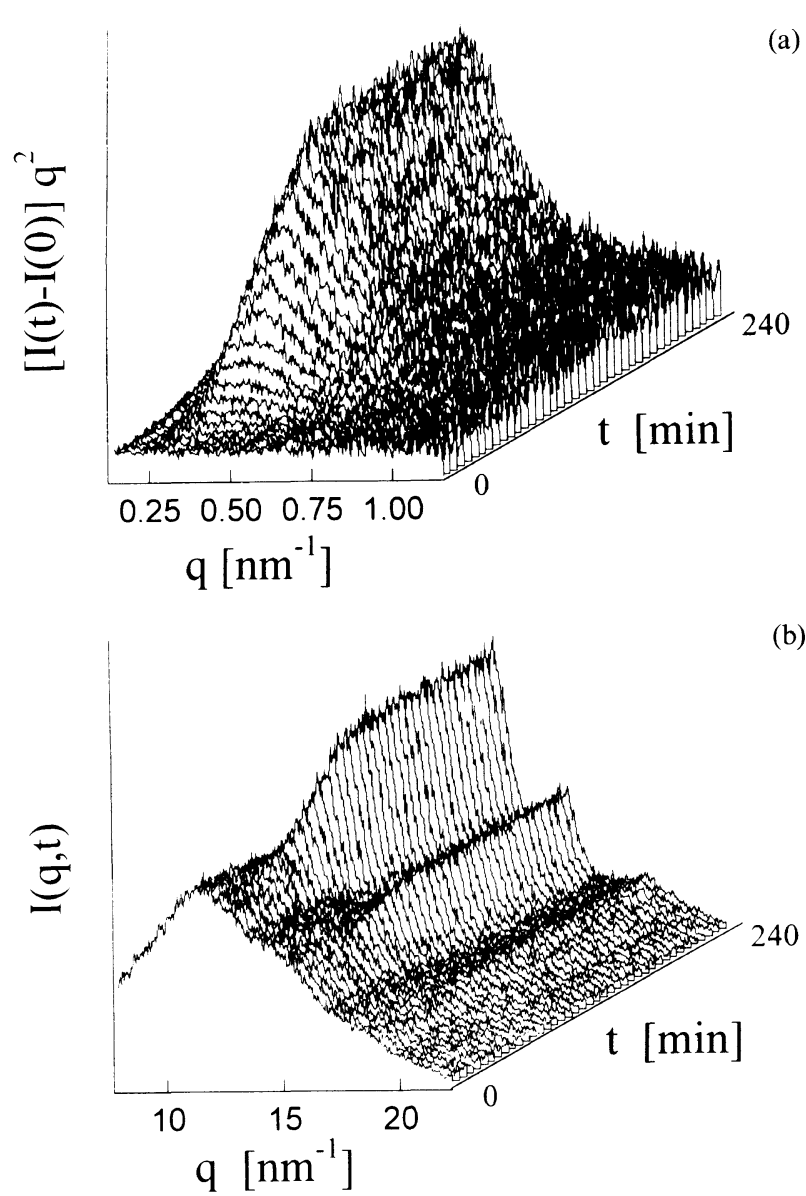

Figure 1. Variation of (a) small- and (b) wide-angle X-ray scattering during isothermal treatment of New-TPI at $T_{\mathrm{c}}=270^{\circ} \mathrm{C}$ from the glass. The patterns were obtained simultaneously using synchrotron radiation.

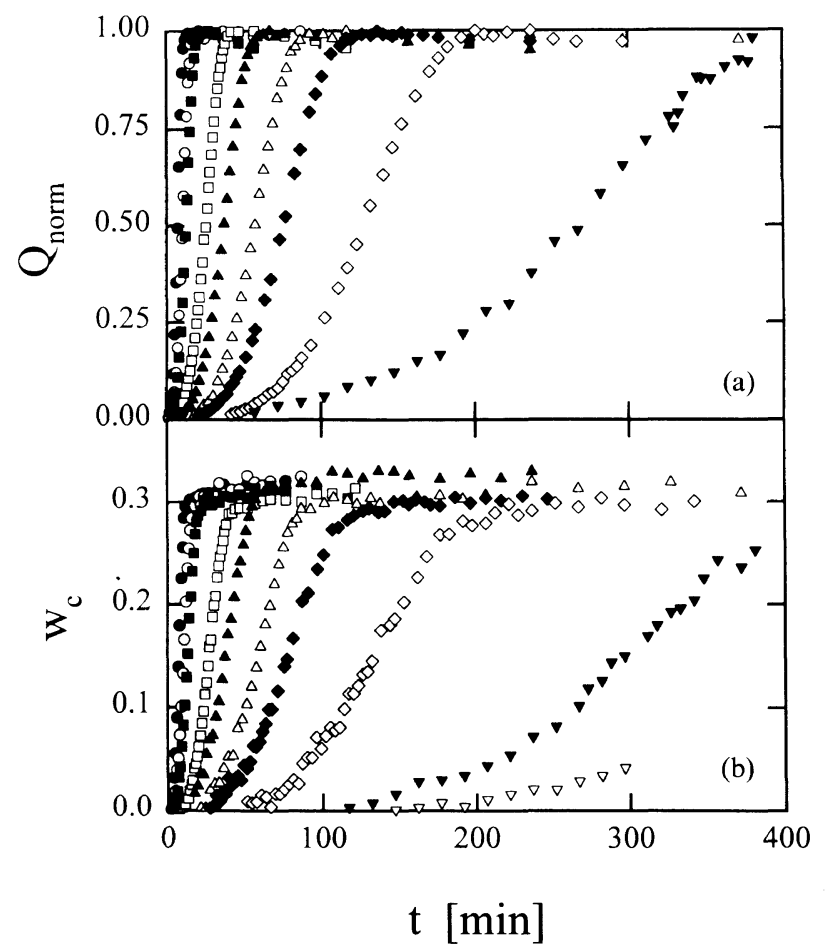

Figure 2. (a) Normalized invariant, $Q_{\text {norm }}$, and (b) relative crystallinity from WAXS, $w_{\mathrm{c}}$, for New-TPI during isothermal crystallization at various temperatures: (from left to right and alternating solid and open symbols) $289,287,285,280,275,272,270,265$, and $262^{\circ} \mathrm{C}$.

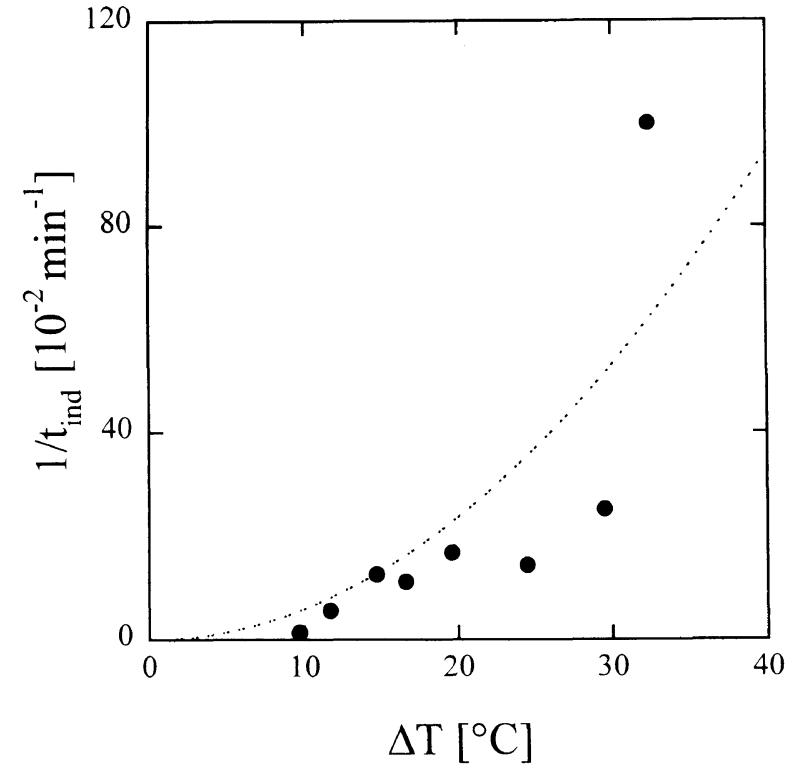

Figure 3. Plot of reciprocal induction time against $\Delta T=T_{\mathrm{c}}-T_{\mathrm{g}}$.

have not been plotted as they could not be normalized). On the other hand the appearance of crystallinity (values larger than $1 \%$ ) is affected by a longer induction period.

The induction time $t_{\text {ind }}$ (difference between appearance of SAXS maximum and WAXS peaks) seems to become longer the lower the crystallization temperature. Figure 3 illustrates the dependence of the reciprocal value of the induction period as a function of $\Delta T=T_{\mathrm{c}}-T_{\mathrm{g}}$ in support of this finding.

\section{Precursors of Crystallization}

The appearance for $T_{\mathrm{c}}=265^{\circ} \mathrm{C}$ of the SAXS maximum before the WAXS peaks suggests that density fluctuations may be a precursor to primary crystallization of New-TPI. Similar behavior has been reported for the semirigid polymers poly(ethylene terephthalate) (PET) and poly(ether ether ketone) (PEEK) crystallized by heating from an amorphous glassy state ${ }^{4,5}$.

Figure 4 shows the time evolution of the logarithm of the normalized integrated SAXS intensity $I(t) / I(0)$ in the range $0.12<\boldsymbol{q}<1.15 \mathrm{~nm}^{-1}$ at $T_{\mathrm{c}}=265,267$, and $270^{\circ} \mathrm{C}$. At the early times of the induction period an increase of $I(t)$ is observed for these isothermal processes (upward arrows in the figure). For times $t^{\prime}>132 \mathrm{~min}$, $t^{\prime}>57 \mathrm{~min}$ and $t^{\prime}>29 \mathrm{~min}$ for $T_{\mathrm{c}}=265,267$, and $270^{\circ} \mathrm{C}$, respectively, the starting of crystallization is characterized by a larger increase of $I(t)$ concurrent to the appearance of Bragg reflections (indicated by arrows pointing downwards).

During the induction period of the isothermal process, $I(t)$ exponentially increases with time at different values of $\boldsymbol{q}$ as shown in Figure 5 for the New-TPI sample isothermally treated at $T_{\mathrm{c}}=265^{\circ} \mathrm{C}$. These scattering data have been analyzed in the formalism described by Cahn and Hilliard ${ }^{10,11}$ in Figure 6. From the intercept with the abscissa a characteristic $\boldsymbol{q}$ value $\boldsymbol{q}_{\mathrm{c}}$ can be estimated from which the most probable wave vector of fluctuations $\boldsymbol{q}_{\mathrm{m}}$ can be derived by using: $\boldsymbol{q}_{\mathrm{m}}^{2}=\boldsymbol{q}_{\mathrm{c}}^{2} / 2$. In our case $\boldsymbol{q}_{\mathrm{m}}(0)=0.5 \mathrm{~nm}^{-1}$. It is noteworthy that this value is similar to that obtained in PEEK and in PET and 


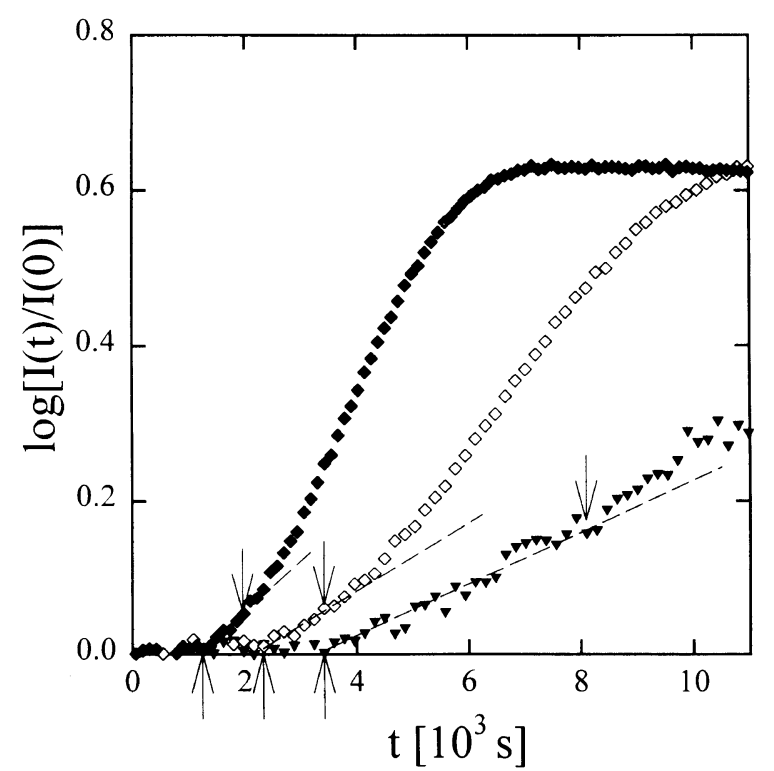

Figure 4. Variation of the integral intensity, $\log [I(t) / I(0)]$, in the range $0.12 \mathrm{~nm}^{-1}<\boldsymbol{q}<1.15 \mathrm{~nm}^{-1}$ as a function of time for various temperatures: $T_{\mathrm{c}}=265(\boldsymbol{\nabla}), 270(\diamond)$, and $272^{\circ} \mathrm{C}(\diamond)$. Downward arrows denote the onset of crystallization. Upward arrows indicate the appearance of SAXS scattering.

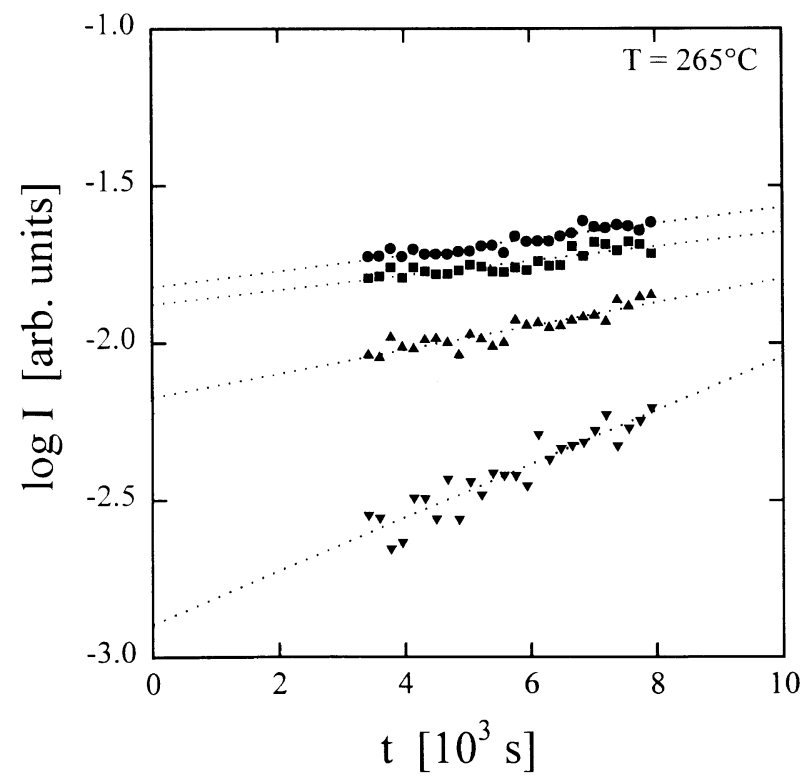

Figure 5. Plot of $\log I$ as a function of time for $T_{\mathrm{c}}=265^{\circ} \mathrm{C}$ and for different $\boldsymbol{q}$ values: $\boldsymbol{q}=0.16(\bullet), 0.23(\boldsymbol{\nabla}), 0.31(\boldsymbol{\Delta})$ and $0.38 \mathrm{~nm}^{-1}(\boldsymbol{\nabla})$.

corresponds to a long range order structure of about $12 \mathrm{~nm}$. Since for $T_{\mathrm{c}}=265^{\circ} \mathrm{C}$ the first measured long period $L$ is about $27 \mathrm{~nm}$ (see Figure 7), this means that the initial fluctuation distance has to increase with time. For higher $T_{\mathrm{c}}$ values a similar treatment does not yield a clear linear plot. This arises the question as to whether such fluctuations prevail at higher crystallization temperatures.

\section{Primary Crystallization}

From the analysis of the WAXS intensity as a function of the crystallization time the Avrami exponent $n$ has been obtained for all samples crystallized at various temperatures. Isothermal kinetics of crystallization is described by a single Avrami exponent $n \simeq 3.2$ suggesting

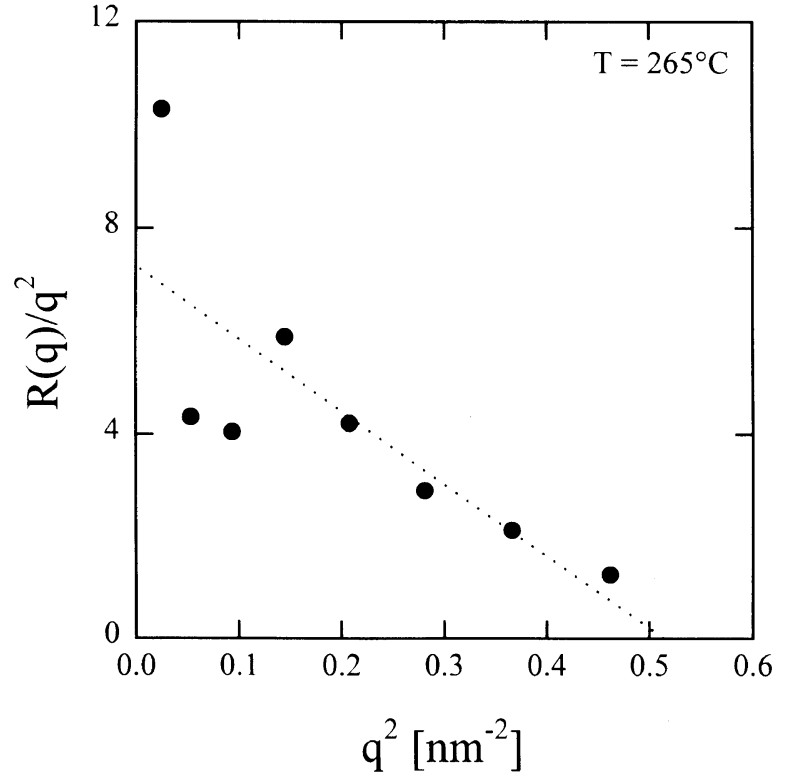

Figure 6. Plot of $R(\boldsymbol{q}) / \boldsymbol{q}^{2}$ as function of $\boldsymbol{q}^{2}$ at $T_{\mathrm{c}}=265^{\circ} \mathrm{C}$ for New-TPI.

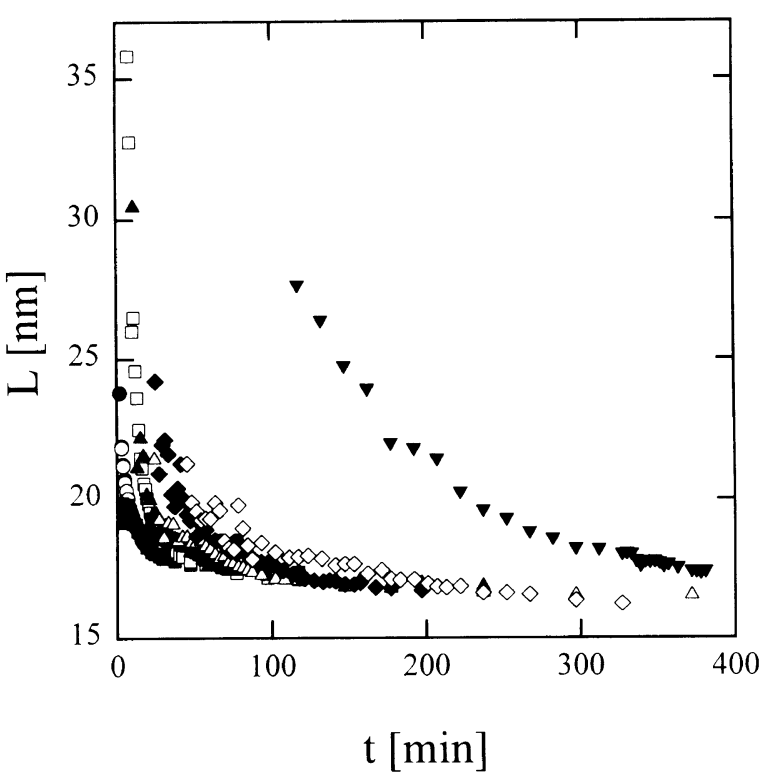

Figure 7. Long period as a function of heating time during cold crystallization at various temperatures (same symbols as in Figure 2).

heterogeneous nucleation process and 3-dimensional growth. These results are in agreement with previous DSC studies. ${ }^{2}$

The calculated long periods, $L$, once the first crystals are formed, have been found to decrease during primary crystallization reaching, in all cases, leveling off values of $\sim 17 \mathrm{~nm}$ (Figure 7). According to the lamellar insertion model the observed decrease may be due to the introduction of thinner lamellae between the preexisting ones. ${ }^{12,13}$ On the other hand proponents of the dual lamellar stack model suggest that the decrease in long period is consistent with the appearance of secondary lamellar stacks having smaller $L$ values as compared to the primary lamellar stacks. At the moment is difficult to decide which one of the two above mechanisms prevails as in both cases $L$ decreases, $w_{\mathrm{c}}$ increases, and the invariant $Q$ remains nearly constant after primary 
crystallization is completed.

\section{CONCLUSIONS}

In conclusion, the analysis of time resolved SAXS/ WAXS experiments suggests that at low $T_{\mathrm{c}}$ values the nucleation step in New-TPI involves a process resembling spinodal decomposition of chain segments with different average conformations. The study of the ordering process when amorphous New-TPI is annealed just above $T_{\mathrm{g}}$ shows that a long range order structure with a size of about $12 \mathrm{~nm}$ is formed during the induction period prior to crystallization. For a temperature $T_{\mathrm{c}}=265^{\circ} \mathrm{C}$, the induction period, where still no crystallization occurs, takes a value as high as $75 \mathrm{~min}$. However, at higher temperatures $\left(T_{\mathrm{c}}>270^{\circ} \mathrm{C}\right)$, the analysis of data does not clearly support the above model.

Acknowledgments. Grateful acknowledgment is due to the International Research Grant NEDO and to the DGICYT, Spain (Grant PB94-0049) for the support of this investigation. Synchrotron radiation experiments were performed thanks to the support of the TMRContract ERBFMGECT 950059 of the European Community. We wish to thank Prof. James C. Seferis, PCL (University of Washington) for the supply of the New-TPI films.

\section{REFERENCES}

1. J. B. Friler and P. Cebe, Polym. Eng. Sci., 33, 587 (1993).

2. P. P. Huo, J. B. Friler, and P. Cebe, Polymer, 34, 4387 (1993).

3. M. E. Cagiao, M. Connor, F.J. Baltá Calleja, and J. C. Seferis, Polym. J., 31, 739 (1999).

4. M. Imai, K. Mori, T. Mizukami, K. Kaji, and T. Kanaya, Polymer, 33, 4451 (1992).

5. T. A. Ezquerra, E. López Cabarcos, B. S. Hsiao, and F. J. Baltá Calleja, Phys. Rev. E., 54, 989 (1996).

6. N. J. Terril, P. A. Fairclough, E. Towns Andrews, B. U. Komanschek, R. J. Young, and A. J. Ryan, Polym. Commun., 39, 2381 (1998).

7. F. J. Baltá Calleja, D. R. Rueda, G. H. Michler, and I. Naumann, J. Macromol. Sci. Phys., B37, 411 (1998).

8. G. Strobl, "The Physics of Polymers," Springer, Berlin, 1996, p 173.

9. Technical Data Sheet A-00, Mitsui Toatsu Chem. Inc., Tokyo, Japan.

10. J. W. Cahn and J. H. Hilliard, J. Chem. Phys., 28, 258 (1958).

11. J. W. Cahn, J. Chem. Phys., 42, 93 (1965).

12. B. S. Hsiao, K. H. Gardner, D. Q. Wu, and B. Chu, Polymer, 34, 3986 (1993).

13. K. N. Krüger and H. G. Zachmann, Macromolecules, 26, 5202 (1993).

14. M. P. Lathmer, J. K. Hobbs, M. J. Hill, and P. J. Barham, Polymer, 33, 3971 (1992)

15. R. K. Verma, H. Marand, and B. S. Hsiao, Macromolecules, 29, 7767 (1996) 